\title{
Three-Phase Distribution Locational Marginal Pricing to Manage Unbalanced Variable Renewable Energy
}

\author{
Lawryn Edmonds, M. Nazif Faqiry, and Hongyu Wu \\ Dept. of Electrical and Computer Engineering \\ Kansas State University, Manhattan, KS
}

\begin{abstract}
This paper investigates three-phase, component-wise real and reactive distribution locational marginal pricing (DLMP). Through the use of case studies, mixtures of positive and negative, as well as real and reactive, DLMP components are explored in detail. A modified three-phase unbalanced 69-node PG\&E system is used to demonstrate the validity of the threephase real and reactive DLMP model. Results emphasize the importance of three-phase pricing schemes and reactive power pricing. The results also indicate the DLMP mechanism can potentially assist in balancing power across phases. DLMP can serve as an economic price signal to efficiently operate the system while minimizing losses, voltage violations, congestion, and imbalances across all phases.
\end{abstract}

Index Terms--distributed energy resources, distribution locational marginal price, reactive power pricing, three-phase unbalanced

\section{INTRODUCTION}

With the development of smart grids and the increase in distributed energy resource (DER) penetration levels, there brings about new challenges to distribution system operators (DSOs). DERs, such as variable renewable energy (VRE), battery energy storage systems (BESSs), conventional generators (CGs), and responsive loads (RLs), increase the complexity of the distribution system. Thus, shifting the characteristics similar to an active transmission system and away from the previously passive system. With an active distribution system, there comes a need for fair and transparent pricing schemes, which reward DERs for reducing losses, voltage violations, congestion, and imbalance of lines. Currently, utilities price electricity demands in the distribution system by using either fixed rates or time-of-use pricing for customers. Also, utilities often use net metering to buy excess energy a residential customer produces at a specified rate. Neither of these practices indicates the price of electricity in real-time. One approach receiving considerable research attention is a pricing mechanism similar to locational marginal pricing (LMP) in the transmission system, referred to as distribution locational marginal pricing (DLMP).

DLMP is defined as the marginal cost to supply the next increment of power to a specific location. It may serve as a price signal for the economic dispatch of generation sources within the distribution system. Incentives arising from nodal

\author{
Ananth Palani \\ Garland Power \& Light \\ Garland, TX
}

pricing may encourage the acceptance of DERs in the distribution system as well as enable the chance for greater revenue of utilities due to reduced losses, voltage violations, and line congestion.

The LMP formulation in the transmission system, which utilizes DC optimal power flow (OPF), cannot be directly used in the distribution system as the resistance to reactance (r/x) ratio is higher than the transmission system and does not reflect the true nodal prices in the distribution system [11]. It remains challenging to use full ACOPF as its non-linear nature does not allow for an efficient way to decompose DLMP into its components, i.e., energy, loss, voltage, and congestion, which are crucial for transactive energy market-based operation of the distribution system. Therefore, linearized ACOPF, considering system topology and constraints, is used to calculate DLMP and its components and has been shown to provide comparable prices to true ACOPF [11]. Linearized ACOPF also immensely reduces the computational complexity of attaining DLMPs.

Along with the idea of nodal distribution pricing, there is a drive for reactive power pricing with the new age of the smart distribution grid. Currently, reactive power is not priced; it is just monitored/penalized if a customer, typically industrial, has a power factor outside of a given range. If reactive power pricing were enabled in the distribution system, it could incentivize reducing reactive power demand and improve power factors.

Unlike the transmission system, the distribution system is somewhat unbalanced. Therefore, there is also a motivation for three-phase nodal pricing. To mitigate imbalances across phases, some utilities monitor the imbalance and manually direct load to other phases after large imbalances occur. Currently, automatic balancing of the system is not practiced. One motive for this research is to use an economic price signal to eliminate imbalance of phases in real-time, while utilizing the maximum amount of VRE possible.

DLMP has recently gained significant research attention [1]-[7], [12]; however, few papers combine real and reactive, component-wise (i.e., energy, loss, voltage violation, and congestion) DLMPs of a three-phase unbalanced system while considering multiple DER types. While [3] provides component-wise real and reactive DLMPs, the effect of the 
unbalanced load and generation case on the DLMP is not considered. Authors in [1] use direct regulation methods, through the use of soft open points, and real power componentwise DLMP signals to solve the congestion management problem in three-phase systems, but do not consider reactive power DLMP. Also, this paper neglects VRE, RL, and BESS models. While [6] models CGs, RLs, and VRE sources, there is no breakdown of the individual DLMP components or reactive power pricing. While [4], [5] model three-phase real and reactive power pricing, there is no component-wise DLMP decomposition. Authors in [2] use state estimation to observe a three-phase unbalanced system with CGs and BESSs. While they break down the DLMP into energy, loss, and congestion components, they ignore the voltage component and reactive power pricing. Authors in [7] provide a component-wise, real and reactive DLMP market model, but ignore VRE, RL, and BESS models. A three-phase ACOPF DLMP model considering DERs and RLs is provided in [12]. Table I summarizes contributions of recently published work associated with DLMPs.

In this paper, we use a real and reactive, three-phase, and component-wise DLMP model accounting for CGs, VREs, RLs, and BESSs [8], to better understand the market-based operation of three-phase unbalanced distribution systems. Through the use of case studies, we show the intricacies between the positive and negative real and reactive energy, loss, voltage violation, and congestion price components making up the total DLMP. We also investigate phase imbalance management using the DLMP mechanism.

TABLE I. Reference Contribution Summary

\begin{tabular}{|l|c|c|c|c|}
\hline \multicolumn{1}{|c|}{ Reference } & $\begin{array}{c}\text { Three- } \\
\text { Phase }\end{array}$ & $\begin{array}{c}\text { Compon } \\
\text { ent-wise }\end{array}$ & $\begin{array}{c}\text { Q } \\
\text { Pricing }\end{array}$ & $\begin{array}{c}\text { VRE } \\
\text { Model }\end{array}$ \\
\hline Bai et al. [3] & & $\checkmark$ & $\checkmark$ & \\
\hline Zhao et al. [1] & & $\checkmark$ & & \\
\hline Wei et al. [6] & $\checkmark$ & & & $\checkmark$ \\
\hline Alsaleh, Liu et al. [4], [5] & $\checkmark$ & & $\checkmark$ & \\
\hline Meng et al. [2] & $\checkmark$ & & & \\
\hline Hanif et al. [7] & $\checkmark$ & $\checkmark$ & $\checkmark$ & \\
\hline Liu et al. [12] & $\checkmark$ & & & \\
\hline This work & $\checkmark$ & $\checkmark$ & $\checkmark$ & $\checkmark$ \\
\hline
\end{tabular}

The remainder of this paper is organized as follows. In Section II, we present the mathematical model of the threephase system and a summary of the linearized three-phase DLMP. In Section III, we provide case studies on the complexities between the DLMP components, and we conclude the work in Section IV.

\section{PROBLEM FORMULATION}

The system model equations and the DLMP decomposition are formulated in the following subsections.

\section{A. System Model}

We assume an unbalanced three-phase system. We index the root node as 0 and order the rest of the nodes from 1 to $\mathcal{N}$, where $\mathcal{N}$ is the total number of nodes/lines. Line $i$ is the line connecting the upstream node, $u(i)$, and the set of downstream nodes, $d\{i\}$, to node $i$. All quantities related to line $i$ are subscripted by index $i$. For example, real and reactive net power injections at node $i$ are denoted by $p_{i}$ and $q_{i}$. Similarly, real and reactive power flows and losses are shown by $P_{i}, Q_{i}, L_{i}^{P}$, and $L_{i}^{Q}$. Superscripts $G, D, B$, and $R$ indicate generation, demand, battery (BESS), and renewable (VRE) energies. Subscripts $i, \phi, t$ index the variable at node $i \in \mathcal{N}$, phase $\phi \in \Phi$, and time $t \in \mathcal{T}$. Here, $\Phi$ is the number of phases, and $\mathcal{T}$ is the number of timeslots. Our objective function is defined in (1), where $C$ is the least cost sum of the day ahead (DA) schedule.

$$
\begin{gathered}
C=\lambda_{t}^{p_{0}} \cdot p_{0, \phi, t}+\lambda_{t}^{q_{0}} \cdot q_{0, \phi, t} \\
+\sum_{i, \phi, w, t} c_{i, \phi, w, t}^{G, p} \cdot p_{i, \phi, w, t}^{G}+\sum_{i, \phi, w, t}^{G} c_{i, \phi, w, t}^{G, q} \cdot q_{i, \phi, w, t}^{G} \\
+\sum_{i, \phi, t} \hat{c}_{i, \phi, t}^{B, p} \cdot \hat{p}_{i, \phi, t}^{B}-\sum_{i, \phi, t} \check{c}_{i, \phi, t}^{B, p} \cdot \check{p}_{i, \phi, t}^{B}+\sum_{i, \phi, t} \zeta \cdot s_{i, \phi, t}^{D}
\end{gathered}
$$

Here, $\lambda_{t}^{p_{0}}, \lambda_{t}^{q_{0}}$ are the real and reactive LMP prices at the substation node, and $p_{0, \phi, t}, q_{0, \phi, t}$ are the real and reactive net power injections at the substation node. If these net power injections are negative, the DSO buys real or reactive power from the distribution system at the respective LMP. CGs bid at a cost of $c_{i, \phi, w, t}^{G, p}$ for real power and $c_{i, \phi, w, t}^{G, q}$ for reactive power, where subscript $w$ indicates the segment energy and bid block using a piecewise bidding method to approximate supply cost of CGs. Variables $p_{i, \phi, w, t}^{G}, q_{i, \phi, w, t}^{G}$ indicate the real and reactive segment power the generators supply. These values must lie within their respective segment constraints and sum to their total output $p_{i, \phi, t}^{G}$ and $q_{i, \phi, t}^{G}$. The reactive power output of the CG must lie within a fraction, $\kappa^{G}$, of the real power output. The BESS units bid at a cost of $\hat{c}_{i, \phi, t}^{B, p}$ for extraction of power from the battery and $\check{c}_{i, \phi, t}^{B, p}$ for injection of power into the battery, where $\hat{c}_{i, \phi, t}^{B, p} \geq \check{c}_{i, \phi, t}^{B, p}$ so the BESS buys power at a low cost and sells at a higher cost to create a positive revenue. Variables $\hat{p}_{i, \phi, t}^{B}$ and $\breve{p}_{i, \phi, t}^{B}$ indicate the power the BESS unit consumes or supplies, respectively. The final term in (1) indicates the value of lost load, $\zeta$, times the sum of the real power demand curtailment, $s_{i, \phi, t}^{D}$ to penalize any load not served.

The total system real and reactive power balance is modeled in (2)-(3), and the real and reactive power net node injection is modeled in (4)-(5).

$$
\begin{gathered}
p_{0, \phi, t}+\sum_{i}\left(p_{i, \phi, t}+L_{i, \phi, t}^{P}\right)=0 \\
q_{0, \phi, t}+\sum_{i}\left(q_{i, \phi, t}+L_{i, \phi, t}^{Q}\right)=0 \\
p_{i, \phi, t}=p_{i, \phi, t}^{D}-p_{i, \phi, t}^{G}-p_{i, \phi, t}^{B}-p_{i, \phi, t}^{R} \\
q_{i, \phi, t}=q_{i, \phi, t}^{D}-q_{i, \phi, t}^{G}-q_{i, \phi, t}^{B}-q_{i, \phi, t}^{R} \\
p_{i, \phi, t}^{D}=\bar{p}_{i, \phi, t}^{D}-s_{i, \phi, t}^{D} \\
0 \leq s_{i, \phi, t}^{D} \leq \sigma^{D} \cdot \bar{p}_{i, \phi, t}^{D} \\
p_{i, \phi, t}^{B}=\hat{p}_{i, \phi, t}^{B}-\check{p}_{i, \phi, t}^{B} \\
p_{i, \phi, t}^{R}=\bar{p}_{i, \phi, t}^{R}-s_{i, \phi, t}^{R} \\
0 \leq s_{i, \phi, t}^{R} \leq \bar{p}_{i, \phi, t}^{R}
\end{gathered}
$$


Equation (6) determines the allocated real demand, $p_{i, \phi, t}^{D}$, from the requested demand, $\bar{p}_{i, \phi, t}^{D}$. The difference between these values, if any, is captured in $s_{i, \phi, t}^{D}$, and is penalized in the objective function as lost load. Equation (7) limits the positive load shedding value to a fraction, $\sigma^{D}$, of the requested demand. Dispatched reactive load is held to $\kappa^{D}$ of the real dispatched load to maintain a constant load power factor.

Equation (8) defines net power extraction of the BESS, $p_{i, \phi, t}^{B}$, which is the difference between the extraction $\left(\hat{p}_{i, \phi, t}^{B}\right)$ and injection $\left(\check{p}_{i, \phi, t}^{B}\right)$ power. The reactive power of the battery, $q_{i, \phi, t}^{B}$, must lie within zero and $\kappa^{B}$ of the real power BESS extraction. Additional BESS constraints defining the state of charge, dis/charging rates, and the minimum number of dis/charging hours can be found in our previous work [9].

Equation (9) determines the dispatched real VRE generation, $p_{i, \phi, t}^{R}$, from the expected generation, $\bar{p}_{i, \phi, t}^{R}$. The difference is captured in $s_{i, \phi, t}^{R}$. Equation (10) limits the positive curtailment value to less than the expected generation. Reactive VRE generation is held to $\kappa^{R}$ of the dispatched real VRE generation.

The real and reactive power flow is defined in (11) and (12), and voltage, $v_{i, \phi, t}$, is defined in (13), where $\mathcal{M}_{i, j}^{P}, \mathcal{M}_{i, j}^{Q}$ are comprised of upstream and downstream incidence matrices and line resistance and reactance diagonal matrices [8]. Note, the real and reactive power loss is approximated using a first-order Taylor series approximation around a feasible point [11]. Equations (14) and (15) enforce voltage and line limit bounds, respectively. Here, $\epsilon$ is some small value, typically $5 \%$, and $\bar{S}_{i, \phi}$ is the maximum allowable MVA line limit. Equation (15) is a linear approximation of the originally convex quadratic apparent power flow limit [10]. Equations (16) and (17) restrict the real and reactive imbalance between phases at the substation node within a small percentage, $\Delta \phi_{p}$, and $\Delta \phi_{q}$. These constraints distinguish this model from our previous singlephase model in [8].

$$
\begin{gathered}
P_{i, \phi, t}=p_{i, \phi, t}+\sum_{k \in d\{i\}}\left(p_{k, \phi, t}+L_{k, \phi, t}^{P}\right) \\
Q_{i, \phi, t}=q_{i, \phi, t}+\sum_{k \in d\{i\}}\left(q_{k, \phi, t}+L_{k, \phi, t}^{Q}\right) \\
v_{i, \phi, t}=v_{o, \phi, t}-\sum_{k \in \mathcal{N}} \mathcal{M}_{i, k}^{P} \cdot\left(p_{k, \phi, t}+L_{k, \phi, t}^{P}\right) \\
-\sum_{k \in \mathcal{N}} \mathcal{M}_{i, k}^{Q} \cdot\left(q_{k, \phi, t}+L_{k, \phi, t}^{Q}\right) \\
1-\epsilon \leq v_{i, \phi, t} \leq 1+\epsilon \\
\left|P_{i, \phi, t}\right|+\left|Q_{i, \phi, t}\right| \leq \sqrt{2} \cdot \bar{S}_{i, \phi} \\
\left|p_{0, \phi, t}-p_{0, \phi^{\prime}, t}\right|=\Delta \phi_{p}, \forall \phi \in \Phi, \forall \phi^{\prime} \in\{\Phi \backslash \phi\} \\
\left|q_{0, \phi, t}-q_{0, \phi^{\prime}, t}\right|=\Delta \phi_{p}, \forall \phi \in \Phi, \forall \phi^{\prime} \in\{\Phi \backslash \phi\}
\end{gathered}
$$

Therefore, the DSO's DA optimization problem formulated in (1)-(17) integrates a real and reactive, three-phase distribution system model to account for CGs, VRE units, RLs and BESSs.

\section{B. DLMP Decomposition}

The linearized DLMP was decomposed in [8] and is summarized here. The real and reactive DLMP is broken down into four components, energy, loss, voltage violation, and congestion costs and is summed to the total real and reactive $\operatorname{DLMP}\left(\Omega_{i, \phi, t}^{P}, \Omega_{i, \phi, t}^{Q}\right)$ as in (18)-(25). Superscripts $E, L, V, C$, $P$, and $Q$ indicate energy, loss, voltage, congestion, real, and reactive components of the total real and reactive DLMP.

$$
\begin{gathered}
\Omega_{i, \phi, t}^{P}=\Omega_{i, \phi, t}^{E_{P}}+\Omega_{i, \phi, t}^{L_{P}}+\Omega_{i, \phi, t}^{V_{P}}+\Omega_{i, \phi, t}^{C_{P}} \\
\Omega_{i, \phi, t}^{Q}=\Omega_{i, \phi, t}^{E_{Q}}+\Omega_{i, \phi, t}^{L_{Q}}+\Omega_{i, \phi, t}^{V_{Q}}+\Omega_{i, \phi, t}^{C_{Q}} \\
\Omega_{i, \phi, t}^{L_{P}}=\Omega_{i, \phi, t}^{E_{P}} \sum_{j} \frac{\partial L_{j, \phi, t}^{P}}{\partial p_{i, \phi, t}}+\Omega_{i, \phi, t}^{E_{Q}} \sum_{j} \frac{\partial L_{j, \phi, t}^{Q}}{\partial p_{i, \phi, t}} \\
\Omega_{i, \phi, t}^{L_{Q}}=\Omega_{i, \phi, t}^{E_{Q}} \sum_{j} \frac{\partial L_{j, \phi, t}^{Q}}{\partial q_{i, \phi, t}}+\Omega_{i, \phi, t}^{E_{q}} \sum_{j} \frac{\partial L_{j, \phi, t}^{P}}{\partial q_{i, \phi, t}} \\
\Omega_{i, \phi, t}^{V_{P}}=\sum_{i^{\prime}}\left(\mu_{i^{\prime}, \phi, t}^{\min }-\mu_{i^{\prime}, \phi, t}^{\max }\right) \frac{\partial V_{i^{\prime}, \phi, t}}{\partial p_{i, \phi, t}} \\
\Omega_{i, \phi, t}^{V_{Q}}=\sum_{i^{\prime}}\left(\mu_{i^{\prime}, \phi, t}^{\min }-\mu_{i^{\prime}, \phi, t}^{\max }\right) \frac{\partial V_{i^{\prime}, \phi, t}}{\partial q_{i, \phi, t}} \\
\Omega_{i, \phi, t}^{C_{P}}=\sum_{j \in u\{i\}} \rho_{i, \phi, t}^{1} \frac{\partial S_{j, \phi, t}}{\partial p_{i, \phi, t}} \\
\Omega_{i, \phi, t}^{C_{Q}}=\sum_{j \in u\{i\}} \rho_{i, \phi, t}^{2} \frac{\partial S_{j, \phi, t}}{\partial q_{i, \phi, t}}
\end{gathered}
$$

The energy components of the DLMP $\left(\Omega_{i, \phi, \mathrm{t}}^{E_{P}}, \Omega_{i, \phi, \mathrm{t}}^{E_{Q}}\right)$ are the shadow prices of (2) and (3). The loss components $\left(\Omega_{i, \phi, \mathrm{t}}^{L_{P}}, \Omega_{i, \phi, \mathrm{t}}^{L_{Q}}\right)$ are found using loss sensitivities with respect to nodal power injections. Similarly, the voltage components $\left(\Omega_{i, \phi, \mathrm{t}}^{V_{P}}, \Omega_{i, \phi, \mathrm{t}}^{V_{Q}}\right)$ are derived using voltage sensitivities to nodal power injections. Here, $\mu_{i^{\prime}, \phi, t}^{\min }, \mu_{i^{\prime}, \phi, t}^{\max }$ are the shadow prices of (14) on all nodes $i^{\prime}$ that hit a lower or upper voltage limit. Finally, congestion components $\left(\Omega_{i, \phi, \mathrm{t}}^{C_{P}}, \Omega_{i, \phi, \mathrm{t}}^{C_{Q}}\right)$ are computed using lines' apparent power flow sensitivities with respect to nodal power injections. Here, $\rho_{i, \phi, t}^{1}, \rho_{i, \phi, t}^{2}$ are combinations of the four Lagrange multipliers that come from the expansion to a linear form of the absolute values in (15), and $S_{j, \phi, t}$ is a combination of real and reactive power injections [8]. When the real or reactive loss, voltage, or congestion components of the DLMP are non-zero at a node, their values, based on dispatch $p_{i, \phi, t}$ or $q_{i, \phi, t}$ of that node, are the sum of the node's marginal contribution to the change in each line's loss, each node's voltage, or the MVA flow of a line, respectively.

\section{CASE STUDY}

A modified 69-node PG\&E system was used to investigate three-phase real and reactive DLMP and its components. A oneline diagram of the system is shown in Fig. 1. Unbalanced loads are indicated using arrows at bus locations. The GAMS platform with a CPLEX MILP solver was used to conduct simulations [13]. We create the following two cases:

Case 1: Typical radial distribution with a low-cost 1.5 MW $\mathrm{CG}$ at Node 62 and no VRE units or BESSs.

Case 2: Heavily unbalanced amount of DERs. CG at Node 62 for all phases. VRE units at Nodes 10, 15, 16, 17, 21, 22, 35, 38, 39, 41, 44, 45, 49, 54, 60, and 64 for Phase A, Nodes 22 and 49 for Phase B, and Node 49 for Phase C. Finally, there is 


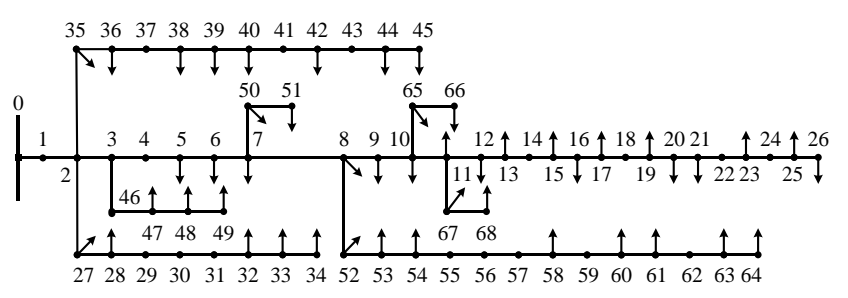

Figure 1. Modified three-phase PG\&E 69-node system.

a 0.5 MWh BESS with minimum charging rate of $0.02 \mathrm{MW}$ and maximum charging rate of $0.05 \mathrm{MW}$ at Node 2 for each phase, of which the DSO has full operational control. This gives a $28.5 \%, 2.6 \%$, and $2.2 \%$ VRE portfolio for Phases A, B, and $\mathrm{C}$, respectively.

\section{A. Impact of Low-Cost CG}

Due to the CG selling at costs much lower than the substation LMP, the CG is producing power until the upper voltage constraint is met. Fig. 2 shows the component-wise three-phase real and reactive DLMP values at Node 8, in which the black line with asterisks shows the total DLMP, i.e., a sum of the component-wise DLMP. Negative voltage components occur on Phases A and B across multiple timeslots. (Most Phase B voltage components are minimal and therefore not visible in the illustration.) Negative voltage components indicate the voltage profile of the distribution system would be improved if more energy were to be consumed at Node 8 at these timeslots. However, the total real power DLMP values are still positive due to the large positive value of the energy component. Congestion occurs in each phase near the peak load hours. The positive congestion component discourages consumption at this node by setting a high price signal. The unavoidable DLMP loss component occurs at every node, but in this case, the values are very small compared to the other components. Therefore, these values are scaled to appear in the visualization.

These results also stress the importance of reactive power pricing. As seen in Fig. 2, real power pricing does not become highly negative in any phase. Reactive power pricing, on the other hand, encounters negative DLMP values. For example, at timeslots 1 and 3-8, Phase A is charging customers a visibly high price to discourage reactive power demand while Phase $\mathrm{C}$ is paying customers to consume reactive power. This is an attempt to meet in the middle, to match the reactive load on Phase $\mathrm{B}$, and balance the reactive power load across all three phases as imposed by (17). Here, $\Delta \phi_{p}=\Delta \phi_{q}=0.2$. A similar balancing result occurs for real power at various timeslots, but the prices do not become highly negative as they do for the reactive power case. The value of reactive power pricing is emphasized here because real power constraints may appear fine while reactive power limits are binding.

There are many timeslots on Phases B and C that appear to be zero in Fig. 2. Some of these timeslots have small DLMP values because the generator supplying the next marginal unit is the low-cost CG. Therefore, the cost to consume the next unit of power is low. In other cases, the CG is at maximum capacity, so the substation is the marginal node serving the next unit of power, thus higher energy component prices.

\section{B. Heavily Unbalanced DERs}

As certain phases can have highly contrasting load and local generation profiles (Case 2), the importance of threephase DLMP modeling is emphasized in Fig. 3. This is a potential future case where one phase of a feeder serves singlefamily homes, capable of widespread rooftop PV, while another serves commercial or apartment complex customers, where rooftop PV is uncommon. Phase A, with high VRE penetration, encounters zero value real DLMPs when the sun is shining, while Phases B and C, with low VRE penetration, do not see zero DLMP values. Here, the zero-variable-cost VRE units are the marginal units supplying the next unit of power. Similar results occur for reactive power pricing. The added local generation in Case 2 helps meet more of the demand than in Case 1, but VRE units are still curtailed. In this case, $23 \%$ of the VRE units are curtailed on Phase A. This result shows the importance of smart DER planning, across phases and locations.

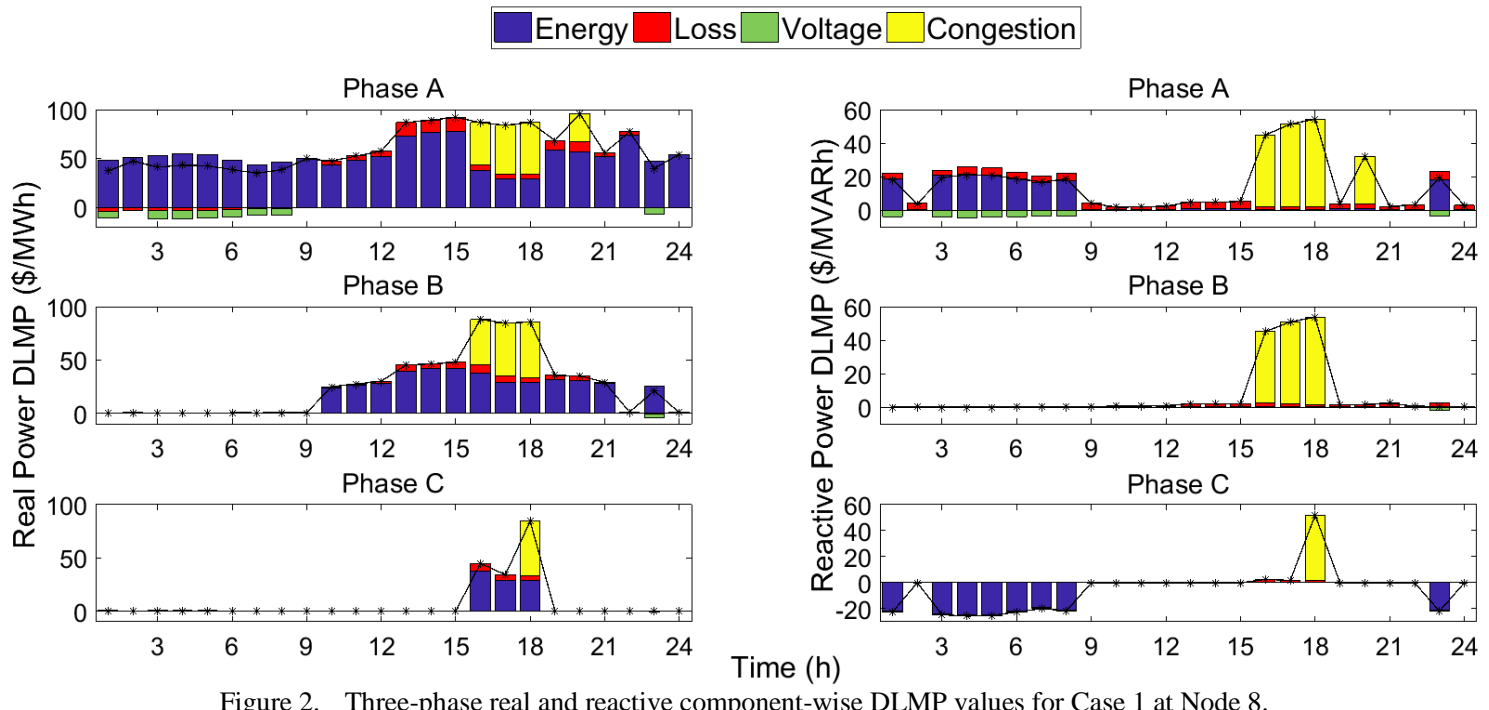

Figure 2. Three-phase real and reactive component-wise DLMP values for Case 1 at Node 8 . 


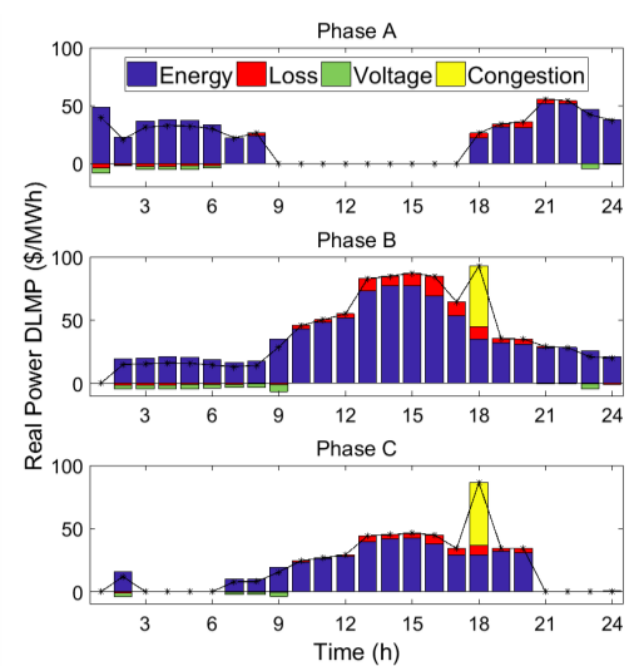

Figure 3. Three-phase real component-wise DLMP values for Case 2 at Node 8 with balance constraints (16)-(17).

During this case, the BESS charges on Phase A at hours 917 when the VRE penetration is high. The BESS on Phases B and $\mathrm{C}$ discharges during these hours to ease the burden of the unbalanced generation profile on Phase A. DLMP values reflect nodal prices in the distribution system, at which the BESS would pay or get paid. If the DLMP is high, the BESS would be paid at that high rate for injecting power, and is thus better incentivized for providing phase balancing services.

\section{Impact of Imbalance Constraint}

To ensure the economic price signal is managing imbalance across phases, we remove constraints (16)-(17) to see the resulting DLMP. The DER parameters are the same as those in Case 2. The proposed model is now minimizing the cost to operate each phase individually, with no coupling between the phases. The results for three-phase real DLMP at Node 8 can be observed in Fig. 4. Here, the energy component across each phase is equal at each timeslot and follows the real power LMP at the substation node. The substation is the marginal node serving the next MW as VRE units are dispatched at full capacity. When comparing Figs. 3 and 4, there is no longer an attempt at balancing the phases using the DLMP mechanism. The energy component is the portion of the DLMP impacted by the imbalance constraint being met. In this case, reactive power energy components are also equal across phases. The results suggest the necessity to consider the threephase balancing constraints in the DLMP model since it provides an inherent mechanism to balance the phases.

\section{CONCLUSION}

To address some challenges brought about by the increased penetration level of DERs, a three-phase, component-wise, and real and reactive DLMP mechanism was formulated and tested on an unbalanced 69-node system. Numerical results show the DLMP mechanism can serve as a tool to balance real and reactive power across phases to encourage/discourage real and reactive power consumption using an economic price signal. It was also demonstrated three-phase pricing allows for better control of diverse DERs in active distribution systems. As shown, real and reactive DLMPs can differ greatly across

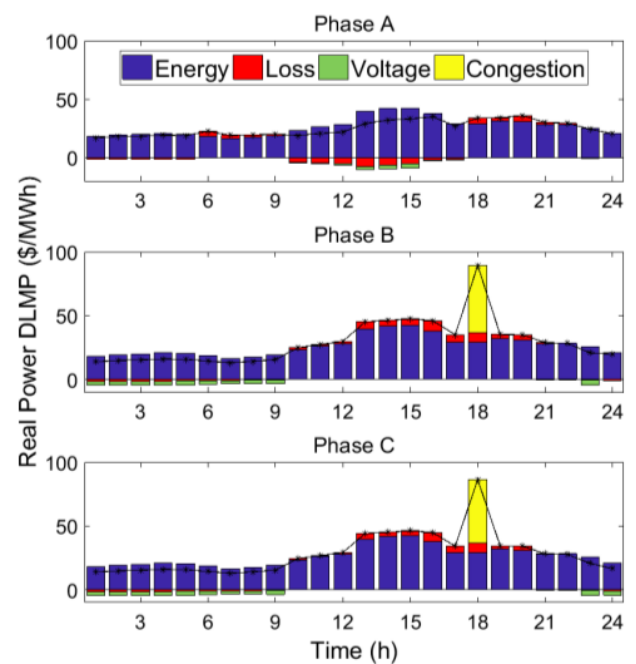

Figure 4. Three-phase real component-wise DLMP values for Case 2 at Node 8 without balance constraints (16)-(17).

phases. Therefore, it is important the DLMP mechanism is able to capture these differences. Interesting future work includes studying the effect of the balancing restriction on the DLMP.

\section{REFERENCES}

[1] J. Zhao, Y. Wang, G. Song, P. Li, C. Wang, and J. Wu, "Congestion Management Method of Low-Voltage Active Distribution Networks Based on Distribution Locational Marginal Price," IEEE Access, vol. 7, pp. 32240-32255, 2019.

[2] F. Meng, D. Haughton, B. Chowdhury, M. L. Crow, and G. T. Heydt, "Distributed Generation and Storage Optimal Control With State Estimation," IEEE Transactions on Smart Grid, vol. 4, no. 4, pp. 22662273, Dec. 2013.

[3] L. Bai, J. Wang, C. Wang, C. Chen, and F. Li, "Distribution Locational Marginal Pricing (DLMP) for Congestion Management and Voltage Support," IEEE Transactions on Power Systems, vol. 33, no. 4, pp. 4061-4073, Jul. 2018.

[4] I. Alsaleh and L. Fan, "Distribution Locational Marginal Pricing (DLMP) for Multiphase Systems," in 2018 North American Power Symposium (NAPS), 2018, pp. 1-6.

[5] Y. Liu, J. Li, and L. Wu, "Distribution System Restructuring: Distribution LMP via Unbalanced ACOPF," IEEE Transactions on Smart Grid, vol. 9, no. 5, pp. 4038-4048, Sep. 2018.

[6] J. Wei, Y. Zhang, F. Sahriatzadeh, and A. K. Srivastava, "DLMP using three-phase current injection OPF with renewables and demand response," IET Renewable Power Generation, vol. 13, no. 7, pp. 11601167, 2019.

[7] S. Hanif, M. Barati, A. Kargarian, H. B. Gooi, and T. Hamacher, "Multiphase Distribution Locational Marginal Prices: Approximation and Decomposition," in 2018 IEEE Power Energy Society General Meeting (PESGM), 2018, pp. 1-5.

[8] M. N. Faqiry, L. Edmonds, H. Wu, and A. Pahwa, "Distribution LMPbased transactive day-ahead market with variable renewable generation," Applied Energy, In Press.

[9] M. N. Faqiry, L. Edmonds, H. Zhang, A. Khodaei, and H. Wu, "Transactive-market-based operation of distributed electrical energy storage with grid constraints," Energies, vol. 10, no. 11, pp. 1891, 2017.

[10] J. A. Taylor, Convex Optimization of Power Systems. Cambridge University Press, 2015.

[11] H. Yuan, F. Li, Y. Wei, and J. Zhu, "Novel Linearized Power Flow and Linearized OPF Models for Active Distribution Networks With Application in Distribution LMP," IEEE Transactions on Smart Grid, vol. 9, no. 1, pp. 438-448, Jan. 2018.

[12] Y. Liu, J. Li, and L. Wu, "Distribution System Restructuring: Distribution LMP via Unbalanced ACOPF," IEEE Transactions on Smart Grid, vol. 9, no. 5, pp. 4038-4048, Sep. 2018.

[13] GAMS Development Corporation. General Algebraic Modeling System (GAMS) Release 28.1.0.; GAMS Development Corporation: Washington, DC, USA, 2019. 